\section{Tecniche costruttive e strutture di potere nella Toscana sud-occidentale (secc. VIII-XIV)}

\author{
GIOVANNA BIANCHI
}

\begin{abstract}
Riasunto
Nell'articolo si tratta l'analisi delle tecniche murarie desunta da dati provenienti da ampi progetti di indagine archeologica svolti in ambito rurale nella Toscana occidentale dalla metà degli anni Novanta dello scorso secolo ad oggi. Nel testo si esaminano $i$ cambiamenti dei modi di edificare a partire dall'edilizia in legno dei primi abitati di altura di vII-VIII secolo, sino agli insediamenti più strutturati di seconda metà VIII e IX secolo, caratterizzati da un primo uso della pietra e dalla presenza di maestranze specializzate. In seguito si analizza la più complessa organizzazione del cantiere propria della costruzione dei castelli di XI e XII secolo in rapporto ai poteri politici ed economici delle nascenti signorie territoriali. Un differente uso delle tecniche costruttive caratterizza la successiva formazione di nuovi borghi tra XIII e XIV secolo, spesso impiantati sui preesistenti castelli, legati ai locali organismi comunali, soggetti all'influenza politica ed economica di Pisa in questo territorio.
\end{abstract}

Parole chiave: tecniche costruttive, edilizia in legno e pietra, organizzazione di cantiere

\begin{abstract}
This paper tells about the analysis of the building techniques of elevations inferred from data obtained in extensive projects of archaeological research executed in western Tuscany's rural field from half ' 90 s of last century to present. The text looks over the changes from wooden building trade related to the first high rise habitants in vith-vilth century, until the pattern settlements in the second half of vilth and xith centuries, characterized by the first use of masonry and the presence of expert master builders. The more complex organization of the building workshops for castles of the xith and xith centuries in relation with the liege lord's rising politic abusive authority is explained in the following part. The subsequent formation of new suburbs between the XIIIth and the xIV centuries is characterized by a different way of use of building techniques, often founded over pre-existing castles, linked to local council, up to the political and economical influence of Pisa in this territory.
\end{abstract}

Key words: building techniques, wooden and masonry building, masonry workshop organization
Il presente lavoro raccoglie una serie di riflessioni incentrate sui modi di costruire in età medievale scaturite dall'analisi dei dati raccolti all'interno di un progetto ricerca con più ampio respiro iniziato dall'insegnamento di Archeologia Medievale dell'Università di Siena, quasi ormai un ventennio fa nel territorio della Toscana sud-occidentale a cui, chi scrive, ha preso parte dall'inizio degli anni Novanta.

Il progetto, ancora in corso, ha come obiettivo lo studio approfondito delle dinamiche insediative nell'area costiera e dell'entroterra della Maremma, sino alle Colline Metallifere, sfiorando i confini dei territori senesi. A questo scopo sono stati avviati, ed in alcuni casi conclusi, estesi scavi all'interno di insediamenti fortificati abbandonati o di complessi monumentali presenti nel tessuto di centri urbani ancora vitali, come campagne di ricognizione di superficie che hanno interessato ampie porzioni del territorio in esame ${ }^{1}$. Contemporaneamente si è svolto lo studio di buona parte delle emergenze in elevato presenti all'interno sia delle aree interessate dagli scavi, sia, in alcuni casi, degli interi centri urbani in cui erano localizzati i depositi scavati (fig. 1).

Se al momento, in tutto il territorio esaminato è stata raggiunta, in relazione alle tecniche murarie, una buona conoscenza dei processi produttivi legati ai secoli XII e XIII, meno informazioni si hanno sui periodi relativi ai secoli centrali ed all'alto medioevo. Un maggiore spiraglio nel cono d'ombra che copre questo arco cronologico, si intravede però nelle aree costiere e nel loro immediato entroterra, dove la ricerca archeologica si è articolata in maniera più capillare, permettendo di cominciare a ricostruire interi contesti sociali, politici ed economici.

Più volte e da diversi ricercatori è stato infatti rimarcato il concetto che la mera campionatura di una tecnica muraria non è sufficiente a comprendere le numerose realtà che stanno dietro alla sua evidenza materiale, se questa non viene ancorata alla complessità dei fenomeni storici che ne hanno determinato la realizzazione ${ }^{2}$.

Dal momento che l'elaborazione di un ambiente tecnico $^{3}$ è una realtà che presenta sfaccettature diverse a seconda dei vari ambiti territoriali, risulta quindi di estrema

\footnotetext{
1 Per i risultati delle ricerche citate si veda Francovich 1991, FranCOVICH-WickHam 1994, BianChi 1995, Belli-De LuCa-Grassi 2003, BIANCHI 2004a; BIANCHI 2004b; BELCARI-BIANCHI 2004, CUTERI 1990; Bianchi 2000; Dallai-Farinelli 1998; Dallai 2000, Casini 2004, Campana-Francovich 2003, Francovich et alii 2000.

2 A questo proposito si veda MANNONI 1988, il capitolo relativo al ciclo produttivo della pietra in MANNONI-GIANNICHEDDA 1996 e BIANCHIFRANCOVICH 2003.

3 All'interno di una comunità, l'ambiente tecnico è costituito da tutte le conoscenze necessarie per presiedere i vari aspetti della vita tecnica. Per la definizione più esaustiva di questo concetto si veda ANGIONI $1984 \mathrm{e}$ BALFET 1981.
} 


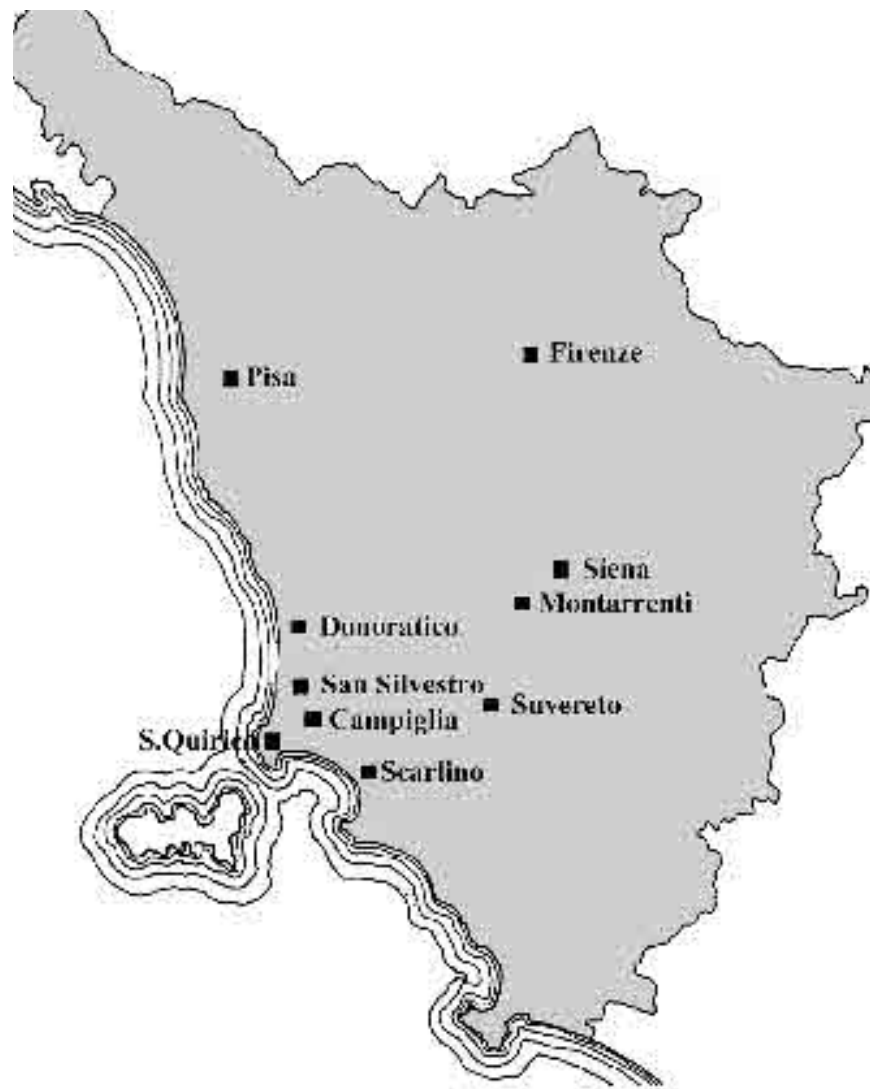

Fig. 1. Nella cartina sono indicati i siti citati nell'articolo oggetti di indagine archeologica

importanza riconoscere queste dinamiche di formazione innanzitutto all'interno di specifici territori, per poi spostarsi ad un ambito regionale o sovraregionale.

L'area costiera e dell'immediato entroterra, su cui ci soffermeremo più specificamente in seguito, rappresenta in questo senso un "osservatorio» di grande importanza, all'interno di una zona geograficamente ben circoscritta e storicamente piuttosto omogenea, nella quale, a fronte di una notevole quanto usuale carenza o totale assenza di notizie documentarie a riguardo, le singole tecniche individuate sono state contestualizzate diacronicamente all'interno delle fasi di evoluzione di singoli assetti abitativi ed agganciate ai tipi di edifici in cui erano utilizzate.

Così facendo, può la tecnica muraria divenire il riflesso di una più complessa realtà economica, politica e sociale legata da un lato alle scelte dei committenti, dall'altro all'opera dei costruttori? Ed è possibile partendo solo dal dato materiale, con pochissimi appoggi delle fonti scritte, tentare di ricostruire le sfaccettature principali di questa realtà?

Partendo quindi dall'analisi di uno specifico territorio, cercheremo di mostrare come questo in parte sia realizzabile, sebbene per tentare una qualunque interpretazio- ne di più ampio respiro vedremo come saranno necessari continui passaggi e collegamenti tra l'evidenza materiale della tecnica e la stessa organizzazione degli spazi abitati, siano essi relativi un singolo monumento od un intero borgo, nel tentativo di relazionare la storia insediativa da un microlivello di lettura (le singole tecniche) ad un macrolivello di interpretazione (le forme di organizzazione politiche e sociali).

Per iniziare questo nostro percorso andiamo quindi intanto a definire le caratteristiche delle committenze.

Da un punto di vista storico tutta l'area fu caratterizzata dall'assenza di grossi agglomerati urbani, elemento che provocò, dalla conquista longobarda, l'interesse di enti che avevano i loro centri lontano da questi territori: la chiesa lucchese, quella pisana, esponenti di famiglie aristocratiche cittadine, organismi monastici. Tra fine del V-VI secolo Populonia sulla costa e Volterra nell'interno, divennero sedi vescovili, andando a definire il territorio di due diocesi confinanti, in cui anche l'influenza dei rispettivi vescovi deve essere considerata all'interno della geografia del potere di questi periodi ${ }^{4}$.

Malgrado fosse evidente da parte degli enti sopracitati la tendenza ad investire energie e capitali nel territorio rurale con l'obiettivo di accaparrarsi il controllo delle diverse risorse, ben poco ci rimane dal punto di vista materiale di questo cruciale momento, tra VI e metà VIII secolo, in cui si cominciano tra l'altro a porre le basi per la formazione di realtà insediative accentrate che precedono l'ordinamento curtense di età carolingia 5 .

Per trovare, le uniche importanti tracce riferibili a questo periodo dobbiamo quindi spostarci nell'interno, ai confini proprio della diocesi di Volterra, dove durante lo scavo del castello di Montarrenti sono stati ritrovati i resti di strutture lignee appartenenti al villaggio di metà VII, seconda metà VIII secolo ${ }^{6}$.

Si trattava di capanne di ridotte dimensioni, di forma rettangolare costruite con pali inseriti in buche di forma circolare, spesso stabilizzati grazie alla realizzazione di rinforzi circolari in terra ed argilla, realizzati intorno al palo dopo il suo inserimento nella buca, secondo una pratica che ritroveremo in seguito anche nell'area costiera. Tra un palo e l'altro l'intelaiatura dei perimetrali era ottenuta con superfici

\footnotetext{
${ }^{4}$ Per l'analisi delle fonti storiche relative a questo periodo si veda CECCARELLI LEMUT 2004.

5 Per una panoramica su questi passaggi storici legata alle evidenze archeologiche, si veda Francovich 2002, FranCOVICH Hodges 2003; VALENTI 2004.

6 CANTINI 2003.
} 
di argilla pressata, terra e ramaglie, seguendo un uso che trova ampi confronti in tutto il territorio toscano e anche nel non lontano villaggio di Poggio Bonizio7.

Da un punto di vista interpretativo, per questi secoli, la lettura delle poche evidenze è quindi piuttosto semplice.

Analogamente ad altri casi dell' Italia rurale centro-settentrionale ci troviamo di fronte ad una realtà tecnica ancora in formazione. Il sapere del costruire in pietra e la capacità di progettare edifici di una certa complessità sono ancora prerogative di un gruppo ristretto di uomini depositari di conoscenze direttamente mutuate dal mondo classico, che si muovono all'interno soprattutto dei cantieri urbani e più raramente, perlomeno in quest'area, nelle zone rurali ${ }^{8}$.

Chi costruisce in questi anni, magari con differenti capacità e perizia, modella il legno per realizzare edifici di limitata ampiezza, con caratteristiche piuttosto semplici, finalizzate sostanzialmente alla risoluzione di problemi di tipo strutturale (solidità della capanna, della sua copertura, infiltrazione di umidità etc.).

E' possibile che questa semplificazione tecnica dipendesse anche dal relativo disinteresse degli esponenti politici, più concentrati sulle risorse territoriali che sulla definizione di precise caratteristiche degli assetti abitativi in quanto riflesso di significativi cambiamenti di potere. In relazione a ciò erano quindi gli stessi abitanti, pur mancando ancora di una precisa percezione che legava ai luoghi il senso di appartenenza ad una comunità, che si organizzavano per realizzare i propri spazi abitativi, codificati poi nell'organizzazione politica del tempo in curtes, vici, casalia come quelli attestati dalle fonti scritte coeve nel territorio da noi esaminato ${ }^{9}$. Abitati spesso già accentrati, localizzati di frequente sulla cima di sommità collinari, costituiti da un gruppo di capanne, difese, come nel caso appunto di Montarrenti, da una palizzata in legno.

E' quindi sostanzialmente dalla seconda metà dell'viII secolo quando, con la conquista franca, il territorio comincia ad essere strutturato in aziende curtensi, che le cose iniziano a cambiare.

Nell'area da noi esaminata la genericità dei luoghi è sostituita da spazi abitati con un proprio nome: le curtes di

\footnotetext{
7 VALENTI 1999; VAlenti 2004. In riferimento ad altri casi toscani per il sito di Terrazzana si veda Quirós CASTILlo 1999. Per ulteriori riferimenti ad altri esempi di edilizia in legno presenti nell'intera penisola si veda l'archivio curato da Fronza in VALENTI 1996 ed i capitoli con relativa bibliografia dedicati alle tipologie costruttive ed ai materiali da costruzione in GALETTI 1998.

8 A questo proposito si vedano le considerazioni relative alla circolazione di queste maestranze in Brogiolo 1984 e 1996, BogRIOLO-GELICHI 1998, GALETTI 1998.

9 Farinelli 2000; Farinelli 2003.
}

Castagneto, Casalappi, del Cornino, di Suvereto, di Scarlino o della stessa Montarrenti, sono forse la spia della rinnovata percezione da parte delle stesse committenze, invariate rispetto al precedente ordinamento politico, che i luoghi, la definizione del loro spazio, la presenza di edifici simbolici potevano rivestire un fondamentale significato nella riorganizzazione non più solo di territori ma anche di quella umanità che li abitava.

L'area costiera come l'entroterra cominciarono ad essere forse maggiormente frequentati dagli stessi rappresentanti del potere centrale o da loro emissari, spesso esponenti delle grandi famiglie aristocratiche, che magari ancora non abitavano stabilmente all'interno delle aziende ma erano incaricati del loro controllo e forse della loro organizzazione insediativa.

Questo passaggio storico è segnato dalla ricomparsa della pietra come materiale da costruzione.

Per questo arco cronologico i dati a nostra disposizione non sono molti, ma restano comunque piuttosto significativi.

A Montarrenti tra seconda metà VIII e prima metà IX secolo, la palizzata in legno che difendeva l'area sommitale è sostituita da una cinta in muratura. Si tratta di un muro costruito con pietre di calcare estratto da fronti di cava molto vicini all'insediamento. Le pietre non presentano nessun segno di lavorazione e finitura e sono poste in opera irregolarmente, legate però da malta di calce.

Per una più completa interpretazione delle vicende legate al cantiere, si sta rivelando decisiva l'indagine archeologica iniziata nell'estate dell'anno 2000 nel castello di Donoratico che, per l'estensione del deposito indagato e per i risultati sinora ottenuti, rappresenta un'importante occasione di arricchimento dei dati e messa a fuoco di ipotesi formulate in precedenti siti già scavati, sulla scorta però di un numero minore di informazioni acquisite dalle evidenze materiali ${ }^{10}$.

In questo insediamento, posto a pochi chilometri di distanza da Castagneto Carducci, dove è attestata una curtis alla metà dell'viII secolo, è stata riportata in luce una chiesa situata all'interno dell'area signorile caratterizzata da tre fasi di ampliamento. Una prima, ascrivibile al IX secolo, che definì un edificio ad unica navata dotato di un'abside; la seconda, avvenuta nella seconda metà dell'XI secolo, caratterizzata dall'ampliamento del perimetro con l'aggiunta di una seconda navata provvista di un'altra abside; una terza, databile al tardo XII secolo, che riguardò l'avanzamento dell'originaria facciata.

\footnotetext{
10 BIANCHI 2004b.
} 
In questo contesto, il dato sicuramente più interessante è il tipo di tecnica muraria utilizzata per la costruzione del primo edificio religioso. Nell'abside ed in parte della navata oggi visibile, si registra infatti l'utilizzo di pietre in locale calcare, che presentano una sommaria squadratura (fig. 2a). Allo stesso tempo alcune delle superfici lapidee, malgrado il loro degrado, sembrano sottoposte ad una spianatura superficiale con uno strumento a punta. I costruttori inoltre disposero i conci seguendo una certa orizzontalità dei filari, cercando di recuperare la non perfetta collisione delle pietre con l'inserimento di zeppe in argilloscisto o frammenti di laterizio di riuso, provenienti dal precedente insediamento di età classica su cui fu costruito il villaggio fortificato ${ }^{11}$.

All'altezza di circa metà della navata una cesura verticale, indicativa di una pausa di cantiere ${ }^{12}$, segna l'impiego di una diversa tecnica muraria, utilizzata anche nell'originaria facciata, con pietre del medesimo calcare però non lavorate e poste in opera irregolarmente, senza seguire nessun filare orizzontale (fig. 2 b). La medesima alternanza di tecniche con le stesse caratteristiche si riscontra pure nei tratti superstiti della cinta muraria stratigraficamente più antica, rispetto ai successivi rifacimenti del circuito difensivo avvenuti nel corso del XII e XIII secolo, che in questi decenni andò a cingere l'intero pianoro sommitale dell'estensione di un ettaro. L'uguaglianza dei modi di edificare proverebbe la contemporaneità delle operazioni costruttive, del resto confermata dai reperti ceramici rinvenuti nei depositi collegati alle strutture murarie in questione. La tecnica irregolare caratterizza anche i paramenti di un imponente muro, coevo al circuito, costruito per segnare una sorta di divisione interna dell'insediamento legata probabilmente all'organizzazione degli assetti produttivi ed insediativi.

Lo scavo archeologico sta gradualmente riportando in luce la presenza, coeva a queste murature, di capanne in legno, spesso di grandi dimensioni e in un caso caratterizzate da un basamento in pietra ed un alzato in legno composto da pali infissi internamente alla base in pietra, portanti una intelaiatura composta da argilla pressata e ramaglie.

Sempre su questo tratto di costa, nella curtis di Scarlino nel corso del IX secolo, si costruisce una chiesa monoabsidata ad unica navata ${ }^{13}$. Dai ridotti resti dei muri perimetrali si nota anche in questo caso l'utilizzo di pietre non

${ }^{11}$ Il perimetro della prima cinta in pietra del castello segue infatti quello del circuito difensivo di una grande fortezza di età etrusca.

${ }_{12}$ La lettura stratigrafica degli elevati e le caratteristiche dei depositi addossa-

ti alle stesse strutture, esclude di interpretare la cesura come traccia di tamponamento o di eventuali rifacimenti.

13 Farinelli-Francovich 2000. lavorate, poste in opera non regolarmente, legate da malta di calce. Questo edificio, in un tempo piuttosto ravvicinato, fu a sua volta racchiuso da un circuito sempre in pietra, esteso lungo il pianoro sommitale, con le medesime caratteristiche tecnologiche dei muri della stessa chiesa.

Nel villaggio sommitale di Campiglia, interpretabile probabilmente come un'azienda gravitante intorno ad uno dei vicini centri curtensi, il fortuito ritrovamento, purtroppo decontestualizzato, di frammenti lapidei provenienti da arredi liturgici, databili tra VIII e IX secolo, attesta la possibile presenza di un edificio religioso in pietra interno o posto a poca distanza dall'insediamento ${ }^{14}$.

Che cosa dedurre quindi da tali evidenze?

La costruzione di una cinta come quella di una chiesa è sicuramente indicativa della sovrapposizione di una progettualità forte, legata ad un attore sociale di una certa consistenza, alla precedente organizzazione degli spazi, con probabilità più strettamente connessa alle esigenze di chi quegli spazi li abitava. Gli antropologi ci insegnano che l'individuo percepisce di appartenere ad un insediamento e quindi alla comunità che lo popola, quando di questo abitato riesce a ricostruire le coordinate spaziali legate a precise strutture materiali, ovvero riesce ad elaborare la «mappa mentale dell'abitato» ${ }^{15}$. Da questo punto di vista le principali categorie spaziali che servono proprio per definire la mappa sono quelle legate alla nozione di limite ed orientamento ${ }^{16}$.

Visto da questa ottica quindi la definizione di un limite e per giunta di una cinta in pietra, probabilmente rivestì a quel tempo un profondo significato perché venne a definire un confine netto tra abitato e non abitato e forse fu uno dei primi passi verso la costruzione di quel senso di identità di appartenenza ad una comunità.

La costruzione della chiesa fu un ulteriore tassello in questa direzione, con duplice valenza. Da un lato infatti la chiesa rappresentava nel nuovo spazio abitato uno dei punti di riferimento fondamentali per la nuova comunità, dall'altro la sua presenza all'interno di un'azienda curtense legata a signorie fondiarie laiche ${ }^{17}$, voleva dire arrogarsi gradualmente di quei vantaggi politici, sociali ed economici sempre gravitanti intorno ad un edificio religioso, oltre che

\footnotetext{
14 BELCARI 2004.

15 LA CECLA 1996.

16 LEVY-SEgaud 1983.

17 In realtà il caso di Donoratico presenta maggiori complessità perché in questa fase di ridefinizione degli assetti il sito poteva essere una delle aziende curtensi del vicino monastero di S.Pietro a Monteverdi, che nel suo patrimonio contava, dal momento della sua fondazione, alla metà dell'vIII secolo, anche la vicina curtis di Castagneto. Solo la prosecuzione dell'indagine archeologica e storica consentirà di definire meglio il ruolo dell'edificio religioso all'interno dell'insediamento, perlomeno tra IX e X secolo.
} 


\section{TECNICHE X SECOLO}

castelli: maestranze specializzate esterne

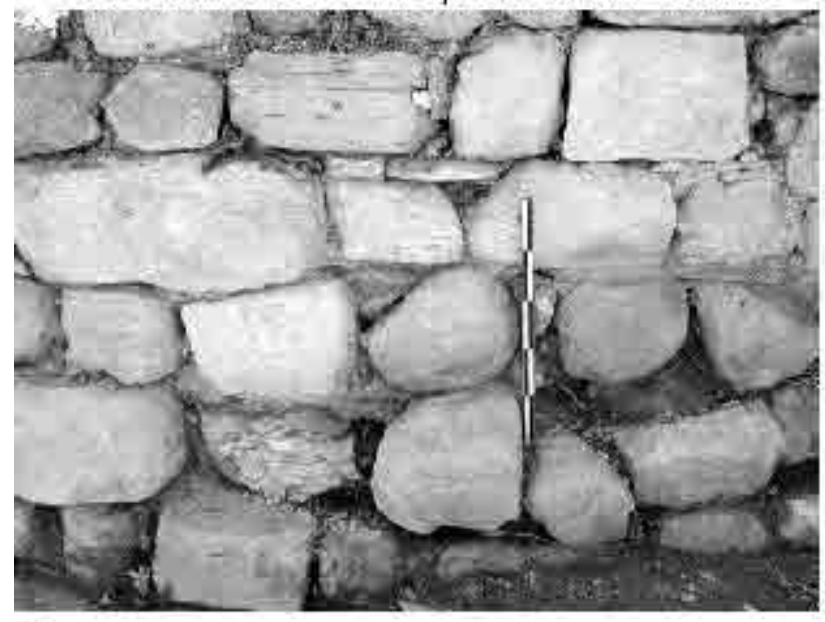

castelli: muratori locali

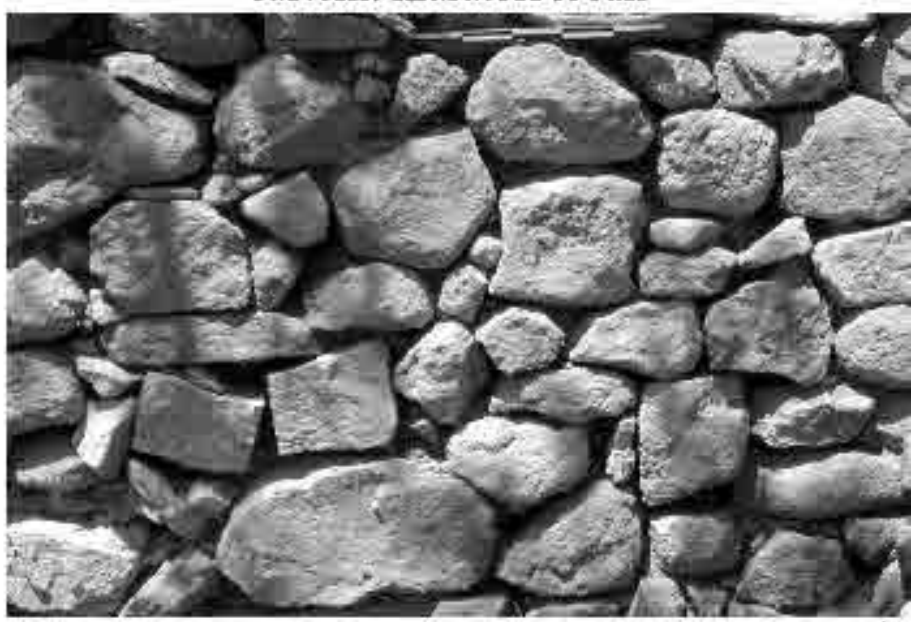

a

TECNICA XI SECOLO

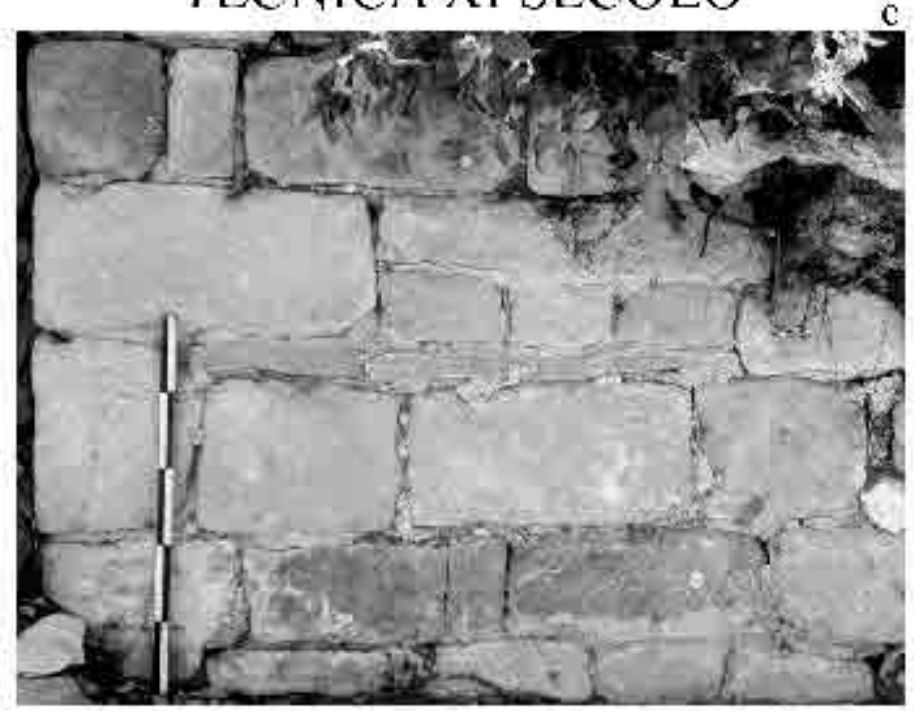

castelli e monasteri:

maestranze specializzate estene

Fig. 2. Esempi di tecniche murarie di x e xı secolo. Per ogni tecnica sono ipotizzate le caratteristiche dei costruttori

emanciparsi e prendere le distanze dagli enti religiosi con cui condivideva il controllo del territorio.

E' quindi evidente che tale realtà insediativa presuppose un'organizzazione del cantiere più complessa e diversa dai precedenti periodi.

Dai documenti, per l'Italia centro-settentrionale, sappiamo della frequentazione saltuaria delle curtes da parte proprio di alcuni esponenti di quei magistri portatori del saper costruire in pietra, che operavano all'interno delle singole aziende, probabilmente istruendo alcuni appartenenti alla comunità che, in seguito a questi passaggi di conoscenze, venivano a loro volta delegati a lavori edili ${ }^{18}$.

Per il territorio in esame possiamo supporre una simile dinamica, con la chiamata da parte delle locali signorie di maestri specializzati a cui fu chiesto di arricchire le aziende curtensi ed il loro territorio di pertinenza, di nuove cinte in pietra ed edifici religiosi.

\footnotetext{
18 Galetti 1997.
} 
Nei documenti è registrato infatti un passaggio di maestri lombardi in quest'area proprio nel corso dell'vIII seco$\mathrm{lo}^{19}$ e sempre dalle fonti scritte sappiamo dell'esistenza di chiese di cui oggi si è persa ogni traccia materiale, la cui costruzione poteva costituire un loro elemento di richiamo.

Nel sito di Donoratico è comunque evidente un'organizzazione del cantiere più articolata.

Qui infatti è provata la coesistenza di due gruppi di lavoro: uno che operava rifacendosi a conoscenze tecniche più specializzate, con un processo produttivo che oltre all'estrazione della pietra e la fabbricazione della calce, comprendeva anche la sbozzatura dei pezzi, pur mancanti di spigoli definiti (con conseguente uso di zeppe litiche per una migliore adesione delle superfici), la sommaria spianatura superficiale e posa in opera abbastanza regolare (fig. 2a): l'altro invece, legato più alla tradizione tecnica diffusa nel territorio $^{20}$, caratterizzato dall'uso di pietre non lavorate, poste in opera iregolarmente (fig. 2b). Come abbiamo visto, l'impiego della tecnica più "curata» la si ritrova di fatto solo in parte della navata e dell'abside della chiesa, mentre in alcuni tratti della cinta si registra ancora la tendenza a mantenere la posa in opera più regolare, senza però in genere provvedere a squadrare la pietra. La tecnica più irregolare è presente ancora in parte della chiesa e nuovamente in buona parte della cinta.

A seguito di queste considerazioni è indubbio che una simile organizzazione del lavoro rimanda sicuramente non a singoli costruttori impegnati in opere di limitata entità, ma ad una committenza pienamente consapevole dei propri progetti, relativi non a singole parti ma alla definizione di un intero contesto abitativo. Una committenza forte, forse legata al vicino centro monastico di S. Pietro in Monteverdi, capace della chiamata di maestranze sicuramente esterne a questo ambiente tecnico che ancora non conosceva la lavorazione della pietra. Maestranze probabilmente provenienti non da altri ambiti rurali ma da un contesto caratterizzato da una certa vivacità di stimoli e circolazione di nuovi saperi.

Se il rapporto di Donoratico con il monastero di S. Pietro a Monteverdi risulterà in futuro l'ipotesi più convincente, è proprio nell'ambito tecnico dei cantieri monastici italiani e d'oltralpe con cui questo monastero era in contatto ${ }^{21}$ che potrebbe essere individuato il serbatoio di queste conoscenze. Altrimenti volgendosi verso ambiti urbani è forse su Pisa, città posta a nord di questo territorio che dobbiamo

\footnotetext{
19 Volpe 1964.

20 Per una panoramica sui modi di costruire nel territorio toscano si veda BIANCHI 2003a.

21 BELCARI-BIANCHI-FARINELLI 2004.
}

incentrare l'attenzione, che sin dall'viII secolo, intratteneva anche una serie di rapporti commerciali con queste aree ${ }^{22}$. In un recente contributo è stata rimarcata la precocità di Pisa nell'uso della lavorazione della pietra, adottata nei più antichi edifici religiosi della città ${ }^{23}$, grazie anche ai suoi articolati contatti secolo con l'intero bacino del Mediterraneo.

E' quindi possibile, analogamente ai secoli successivi, che da qui partissero o si formassero quelle maestranze poi circolanti nei territori rurali posti nell'area maremmana, richiamate da una situazione politica estremamente favorevole per la presenza di committenze forti, impegnate a costituire i propri poteri grazie anche alla realizzazione di rinnovati insediamenti fortificati.

E' possibile inoltre che il gruppo di specialisti attivi a Donoratico fosse numericamente ristretto, vista l'adozione di questa particolare tecnica solo in alcuni punti del sito (la chiesa appunto e parti della cinta). Per la realizzazione delle strutture di tutto l'insediamento fu quindi necessario l'aiuto di muratori operanti già nel luogo di origine, provvisti di un bagaglio tecnico formatosi e tramandato in loco. Il dato comunque interessante, oltre appunto la chiamata di muratori esterni, è in ogni caso la coesistenza delle tecniche, anche nel medesimo edificio, segno che in corso d'opera non vi fu poi un così consistente scambio di saperi tra i due gruppi di lavoro, con la conseguente creazione di tendenze tecniche tra i muratori locali. Ognuno dei due gruppi operò in sintonia rifacendosi però alle proprie conoscenze.

Una giustificazione alla compresenza di due tecniche così evidentemente differenti potrebbe ritrovarsi nell'uso di un'intonaco parzialmente coprente le superfici a vista, che di conseguenza avrebbe anche limitato l'impatto visivo di apparecchiature diversificate ${ }^{24}$.

A parte quindi il caso di Donoratico, dalle caratteristiche tecniche delle muratura sia delle nuove cinte sia degli edifici religiosi è evidente che le conoscenze di queste maestranze non riguardavano per il momento la squadratura o la parziale lavorazione della pietra. In ogni caso il processo produttivo comprendeva l'estrazione dei pezzi dal fronte di cava e la fabbricazione della calce. L'esistenza dei frammenti lapidei di arredo con caratteristiche stilistiche di un discreto livello, come nel caso di Campiglia, attesta inoltre la circolazione di scultori capaci, provvisti di strumenti per la finitura della pietra, non utilizzati però nei muri perimetrali.

\footnotetext{
22 Ceccarelli 2004.

23 Quirós Castillo 1998.

24 Tracce di questo intonaco, che copriva i limiti della pietra lasciando parzialmente a vista il centro delle superfici, sono state infatti identificate in alcuni lacerti dei muri della chiesa e della cinta.
} 
Le indagini archeologiche hanno inoltre evidenziato la presenza coeva a queste strutture in pietra, di capanne in legno, indicative dell'uso puntuale del materiale lapideo solo nelle più importanti e rappresentative parti dell'insediamento.

Le esigue tracce materiali non consentono però di verificare ulteriori caratteristiche dell'organizzazione del cantiere, ben più evidenti per il successivo periodo, corrispondente al X secolo.

In questo secolo qui, come nel resto della Toscana, si iniziano infatti ad intravedere quelle consistenti trasformazioni che preluderanno al complesso fenomeno dell'incastellamento ${ }^{25}$.

Le fonti scritte ci tramandano l'immagine di un'organizzazione territoriale ancora legata all'ordinamento curtense, che in questo territorio sembra perdurare sino perlomeno all'XI secolo ${ }^{26}$.

Nella definizione di questi luoghi abitati che caratterizzavano la campagna toscana, comincia però a comparire, giustapposto alla curtis, il termine castello, con una frequenza sempre più elevata mano a mano che ci si sposta verso la seconda metà del $\mathrm{X}$ secolo ${ }^{27}$.

$\mathrm{E}^{\prime}$ indubbio quindi che qualche cosa stava cambiando ad un ritmo piuttosto sostenuto, in relazione soprattutto alla definizione di nuovi poteri.

Nel territorio costiero ad esempio, come scrivevamo sopra, si passa dall'attestazione di curtes nel corso dell'vIII e IX secolo a documenti che all'inizio dell'XI secolo (1004), in occasione di un atto di donazione, ci informano non solo della presenza di un'importante famiglia aristocratica in questo territorio, i Della Gherardesca, di cui sino ad allora non avevamo nessun notizia, ma del controllo che questa casata aveva di una serie di insediamenti posti in quest'area, codificando in tal modo due precise realtà: l'esistenza di siti fortificati già definiti castelli e l'avvenuta formazione di un consistente patrimonio legato ad un gruppo familiare ${ }^{28}$.

Certamente il caso dei Della Gherardesca è uno dei più evidenti riguardò alla repentinità di formazione di una signoria territoriale, ma non si discosta molto ad esempio da quello dell'altra importante famiglia, gli Aldobrandeschi, a cui sono invece legati molti castelli nell'area costiera più a sud e dell'entroterra. Se infatti la presenza degli Aldobrandeschi, provenienti da Lucca, è registrata in questa zona già dall'altomedioevo, è comunque altrettanto rapido, nel loro caso, il passaggio tra X ed XI secolo dal controllo di curtes alla gestione di centri già incastellati ${ }^{29}$.

Il $\mathrm{X}$ secolo, quindi, in questo territorio sembrerebbe segnare una fase decisiva, in cui alcuni esponenti delle famiglie sopracitate, magari inizialmente investiti dai principali enti che controllavano la zona, di funzioni pubbliche di carattere amministrativo, relative alle aziende curtensi, gradualmente cominciarono ad esercitare su questi insediamenti un controllo sempre più capillare ed esclusivo.

Visto però che le fonti scritte, riguardo a questo cruciale momento, scarseggiano di indizi, quali dati sono desumibili dalle evidenze materiali?

In base a queste si deduce per il X secolo sicuramente un uso molto più diffuso della pietra, che comincia ad essere utilizzata nella maggior parte degli insediamenti conosciuti.

Se lo scavo dell'area sommitale del castello di Campiglia Marittima, ci ha recentemente restituito l'immagine di un villaggio nel $\mathrm{X}$ secolo ancora legato ad un'economia sostanzialmente rivolta all'autoconsumo, ancorata ad attività silvo-pastorali ${ }^{30}$, nella seconda metà del $\mathrm{X}$ secolo, ad esempio, qui, fu costruito, poggiato sui livelli di vita di una precedente capanna in legno, un edificio in pietra di cui sono stati individuati solo pochi lacerti, sufficienti comunque per leggere l'utilizzo di pietre non lavorate, poste irregolarmente ma legate da malta di calce anche di buona qualità ${ }^{31}$. Anche nel vicino castello di Rocca San Silvestro, alla fine del X secolo, inizio di quello successivo, furono costruite due cinte in pietra (sommitale ed a recinzione del borgo) $\mathrm{i}$ cui ridotti tratti superstiti mostrano affinità con la tecnica utilizzata a Campiglia, trattandosi sempre di murature con pietre non lavorate, in ambedue i casi estratte da cave vicine od interne allo stesso sito, legate da malta di calce ${ }^{32}$ (fig. 2b). Caratteristiche che comunque non sembrano mostrare grosse differenze con le murature in pietra più antiche, di cui abbiamo scritto in precedenza riguardo ai siti di Montarrenti, Scarlino e parte dei muri di Donoratico.

Quindi due insediamenti vicini, Campiglia e Rocca San Silvestro, ambedue definiti castelli nell'xi secolo e legati ai Della Gherardesca, con destini formativi diversi: il primo, dove la fortificazione si impianta su di una preesistente realtà abitativa risalente perlomeno al IX secolo; il secondo, di nuova fondazione che sembra essere definito proprio tra la fine del X-inizi XI secolo. In tutte e due i casi è evidente l'impiego di una tecnica muraria caratterizzata da pietre non

\footnotetext{
29 Per una generale ricostruzione degli eventi storici riguardanti questa famiglia si veda CollaVINI 1998.

30 BIANCHI 2004c.

31 BIANCHI $2004 \mathrm{~d}$.

32 BIANCHI 1995.
} 
lavorate, poste in opera irregolarmente ma legate da malta di calce (fig. 2b).

Certo, la specializzazione delle stesse maestranze esterne era ancora lontana da quella degli scalpellini che lavoreranno nel territorio a partire dal XII secolo, in grado di rifinire perfettamente gli spigoli dei conci. Così forse come era ancora lontano nel tempo il significato simbolico della perfetta squadratura della pietra in edifici civili o religiosi pri$\mathrm{vi}$, in seguito, di intonaci di rivestimento perlomeno nei paramenti esterni ma è con queste premesse tecnologiche, che l'XI secolo, rappresentò un importante periodo di passaggio, in questo come nei territori limitrofi.

In contemporanea alla sempre più graduale crescita di importanza politica e sociale dei centri fortificati ed all'ormai più consistente flusso di maestranze itineranti, impegnate oltre che nella costruzione di castelli, anche in quella di monasteri e chiese o pievi, l'uso della pietra nell'XI secolo cominciò a diffondersi infatti anche per l'edilizia abitativa.

Le capanne costruite totalmente in legno a Scarlino, Montarrenti e Campiglia, furono sostituite da altre con il medesimo perimetro rettangolare e simile estensione, ma costituite da un basamento con pietre non lavorate, legate da malta di argilla e terra, con un alzato in legno ${ }^{33}$. L'ambiente era poi coperto da un tetto in paglia a doppio spiovente, sorretto da pali interni infissi in buche circolari con rinforzi circolari in argilla e terra, simili a quelli evidenziati nelle fasi più antiche di Montarrenti e Donoratico. La relativa semplicità delle strutture, come lo stessa scelta dei materiali da costruzione sembrerebbero rimandare al lavoro di costruttori-abitanti che operavano in loco. La presenza però di alcuni elementi simili in insediamenti anche distanziati, come il perimetro rettangolare e soprattutto l'uso di rinforzi ai pali parrebbe invece legata ad un patrimonio di conoscenze comuni spiegabile solo forse con il contatto tra i vari costruttori.

Se accanto a queste capanne, malgrado la citazione in pochi documenti di X ed XI secolo di torri ${ }^{34}$, non sono stati ancora individuati strutture con caratteri propriamente signorili, è ancora negli edifici religiosi che si notano i graduali passaggi tecnologici dovuti al lavoro di maestranze con un più approfondito bagaglio di saperi.

Nell'ampliamento della chiesa di Donoratico, come nella costruzione della chiesa a tre absidi del monastero di S. Quirico ${ }^{35}$, infatti è ben evidente la maggiore cura nella lavorazione della pietra che portò alla fabbricazione di conci

BIANCHI 2004d

34 Augenti 2000.

35 Per i primi dati sullo scavo nel monastero di S. Quirico si veda FrANCOVICH-GELICHI c.s. con spigoli meno arrotondati e maggiormente squadrati, accompagnati da una maggiore precisione nelle posa in opera, essendo necessario un minore uso di zeppe per la migliore aderenza tra le singole pietre. In percentuale fu inoltre maggiore il numero delle superfici a vista spianate con uno strumento a punta (fig. 2c).

$\mathrm{Si}$ trattò in ogni caso di importanti tappe tecnologiche che andarono di pari passo con l'infittirsi degli scambi commerciali tra questo territorio ed in particolare $\mathrm{Pisa}^{36}$, necessarie ad arricchire l'ambiente tecnico delle singole comunità in vista dei grandi cambiamenti di XII secolo.

In questo periodo infatti, nel territorio in esame, come anche in tutta la Toscana, il definitivo riconoscimento della signoria territoriale ed il suo acquisito peso politico ed economico si tradusse nella riorganizzazione di buona parte dei preesistenti siti fortificati.

L'area costiera e dell'entroterra divennero una sorta di grande cantiere aperto dove operavano più gruppi di maestranze addette alla progettazione di nuove cinte in pietra, edifici signorili, chiese ma anche di case del borgo racchiuso all'interno del circuito difensivo, ora edificate totalmente in pietra.

Data l'entità delle ricostruzioni, si trattò, per ogni sito, di progetti impegnativi con l'obiettivo principale di definire materialmente una realtà insediativa che oltre a svolgere la funzione di centro produttivo e di raccolta della popolazione, doveva anche rappresentare materialmente l'acquisizione dei nuovi poteri.

In questa ottica, la tecnica muraria ed in particolare la squadratura della pietra, in parallelo a quanto avvenne per le architetture religiose, acquisirono un valore simbolico aggiunto. Scomparsi, perlomeno nelle superfici esterne, gli intonaci di rivestimento, i nuovi paramenti murari divennero in questo contesto storico una sorta di manifesto politico, una precisa spia della topografia dell'insediamento ${ }^{37}$.

L'intero processo produttivo non fu frammentato in tempi differenziati ma avvenne in contemporanea, sotto lo stretto controllo dei locali signori o dei loro affiliati.

Gruppi di maestranze qualificate, ora capaci di squadrare perfettamente la pietra, coordinarono un più ampio gruppo di muratori locali (fig. 3a-b-c). La necessità legata alla precisa volontà signorile, di costruire un insediamento con caratteristiche tecniche non differenziate, portò però in

\footnotetext{
36 I dati riguardanti lo studio della ceramica proveniente dallo scavo della Rocca di Campiglia, attestano già da questo periodo l'infittirsi di rapporti tra questo territorio e la città, GRASSI 2004.

37 BIANCHI 1995; BIANCHI 1996.
} 


\section{TECNICHE XII SECOLO}

castelli: lapidici e muratori specializzati

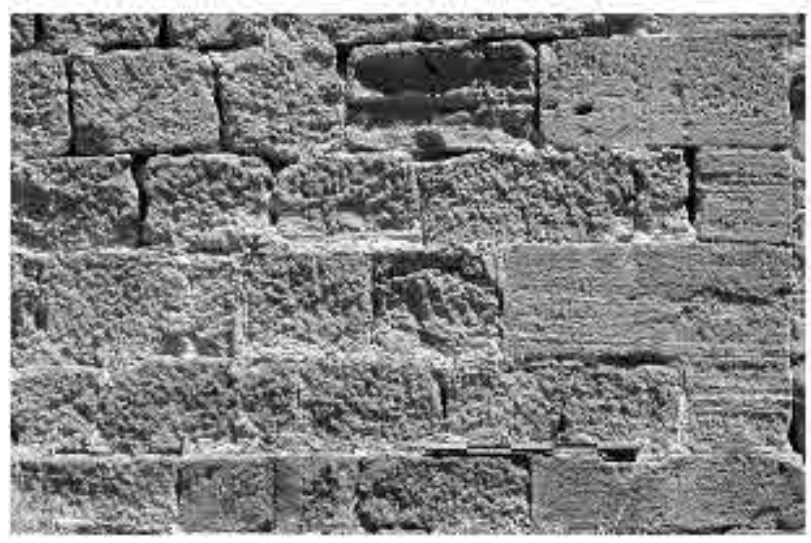

a castelli: muratori locali

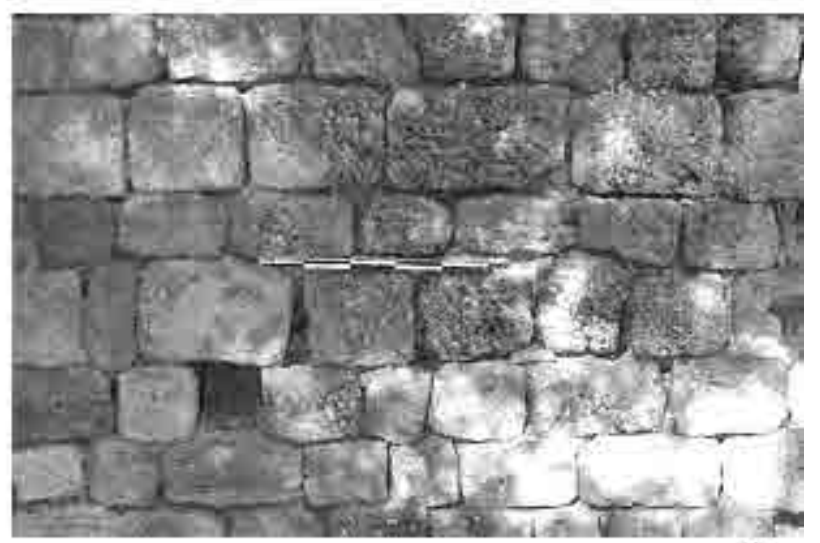

b

chiese:lapidici specializzati

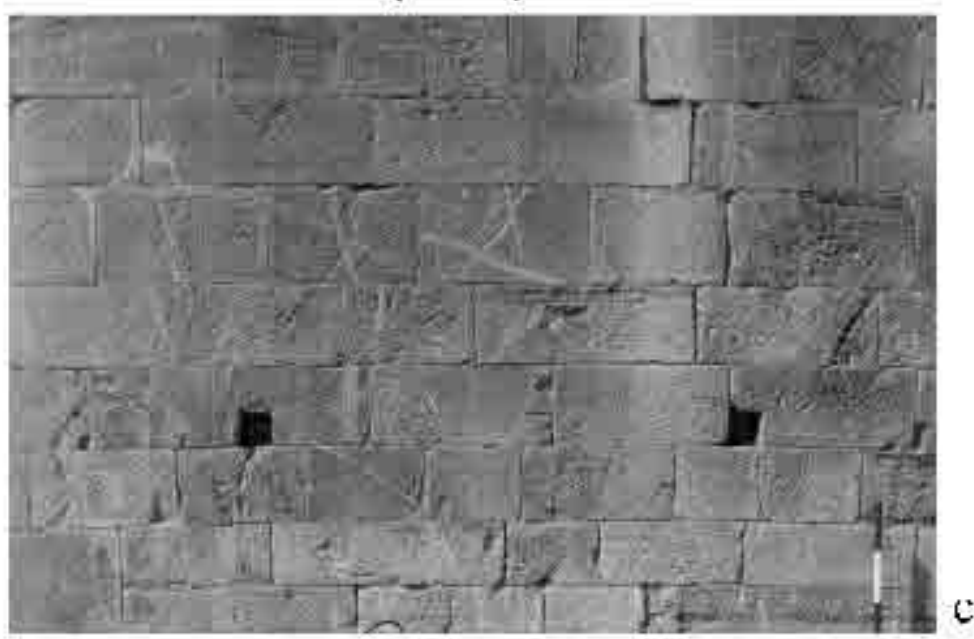

\section{TECNICHE XIII SECOLO}

castelli: muratori-abitan1i

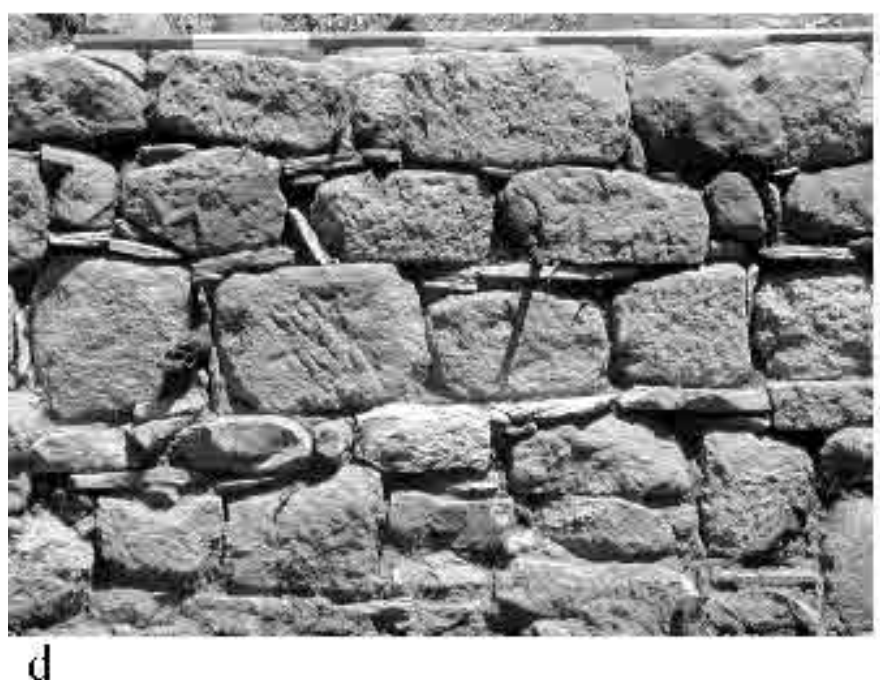

borshi comunali: muralori specialil\%/ali cstlerni

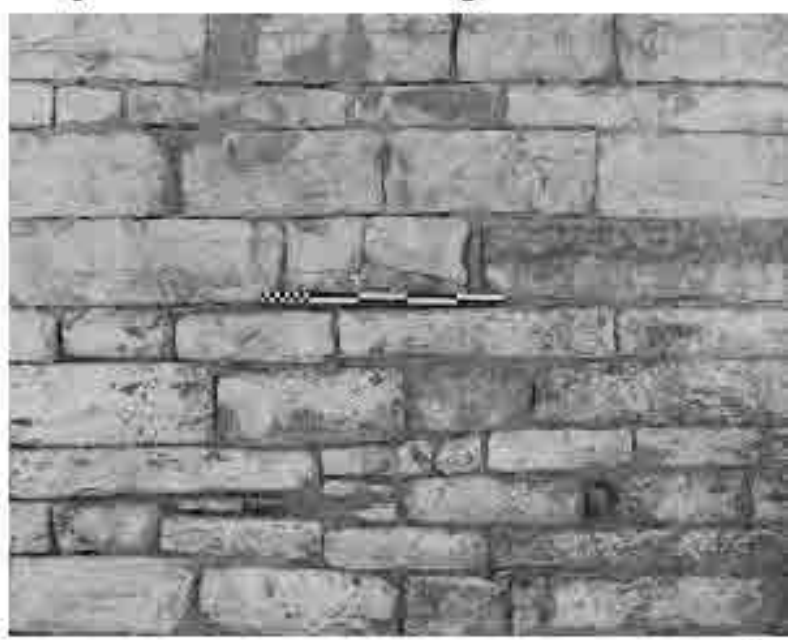

$\mathrm{e}$

Fig. 3. Esempi di tecniche murarie di XII e XIII secolo. Per ogni tecnica sono ipotizzate le caratteristiche dei costruttori 
questa fase ad una trasmissione veloce ed in certi sensi forzata di conoscenze tecniche.

Da un punto di vista materiale questi passaggi tecnologici sono riscontrabili nella comparsa di una tecnica muraria che non ha più nulla a spartire con quella legata alla cultura costruttiva locale, caratterizzata, ricordiamo, dalla non lavorazione della pietra e dalla posa in opera irregolare.

Agli scalpellini fu infatti affidato il compito di realizzare interamente od alcune parti degli edifici più rappresentativi dell'insediamento: la residenza signorile, la chiesa, i più importanti tratti del circuito difensivo (ad esempio dove si apriva l'unica porta di accesso al castello). Al più numeroso gruppo di muratori locali fu riservata invece la costruzione delle altre parti del sito, le case del borgo, la maggioranza del circuito difensivo. La spia materiale indicativa del lavoro dell'uno o dell'altro gruppo di costruttori è data da significative differenze nella tecnica muraria adottata. Quella degli scalpellini è infatti contraddistinta da una perfetta squadratura della pietra con rifinitura della superficie interna a vista con subbia e scalpello nell'anatirosi del concio (fig. $3 \mathrm{a}-\mathrm{c}$ ). Le pietre sono disposte seguendo un'ordinata posa in opera, senza zeppe in pietra, con giunti e letti di posa di limitate dimensioni.

La tecnica applicata dai locali si caratterizza invece per la presenza di spigoli del concio in genere non definiti con esattezza e per una spianatura superficiale più sommaria con solo l'utilizzo della subbia e non dello scalpello. In relazione al tipo di lavorazione delle pietre, la posa in opera è meno regolare con l'inserimento frequente di zeppe in pietra e realizzazione di filari sdoppiati (fig. 3b).

Una simile organizzazione è quindi interpretabile come una piccola rivoluzione all'interno di un ambiente tecnico locale ben assestato su procedimenti costruttivi ormai tramandati empiricamente da quasi un secolo e mezzo, di generazione in generazione. In tale ambiente l'arrivo di maestranze altamente specializzate di cui, per espressa volontà signorile, si doveva seguire il lavoro, rappresentò indubbiamente la rottura di quella continuità strumentaleconoscitiva propria di buona parte delle comunità.

Ciò portò all'elaborazione da parte dei muratori locali non di una nuova tecnica, ma di un modo di costruire simile ma non uguale a quello delle maestranze specializzate. Ancora una volta gli antropologi ci dicono come la risultante di stimoli tecnici esterni alla comunità con quelli interni elaborati dal gruppo, produce la cosidetta «tendenza tecnica», ovvero il grado di rielaborazione dello stimolo stesso da parte dei singoli operatori della comunità ${ }^{38}$. Solitamente i

38 LEROI-GOURHAN 1971. tempi di rielaborazione sono abbastanza ampi e sono legati ad un insieme di fattori interni al gruppo (conoscenze tecniche, strumenti a disposizione etc.).

Se quindi l'elaborazione della tendenza tecnica riguardante i primi muri in pietra, tra IX e X secolo, avvenne secondo tempi più dilungati che permisero forse la graduale acquisizione e diffusione delle nuove nozioni da parte di alcuni membri delle comunità divenendo parte integrante dei singoli ambienti tecnici, per il XII secolo i passaggi si verificarono in maniera differente.

Come scrivevamo sopra, tutto si risolse in un processo costruttivo piuttosto velocizzato, in cui gli abitanti costruttori non ebbero in definitiva tempo di assorbire appieno le nuove nozioni, ma si limitarono a seguire, imitare, il lavoro degli specialisti senza però che questo divenisse realmente patrimonio comune dell'ambiente tecnico delle singole comunità.

Il risultato di questi avvenimenti sono appunto tecniche murarie vicine ma non uguali a quelle degli scalpellini e l'immediata riappropriazione, in tempi successivi al XII secolo, del modo di costruire conosciuto.

Prima però di andare a parlare dei secoli successivi, soffermiamoci ancora un poco su questo importante periodo.

Riepilogando quanto sinora detto, in buona parte del territorio la ricostruzione dei castelli nel XII secolo avvenne quindi grazie al lavoro congiunto di un gruppo ristretto di scalpellini, portatori di nuove conoscenze (in particolare relative alla lavorazione della pietra ${ }^{39}$, aiutati sul campo da un più numeroso gruppo di muratori locali, che abitava nell'insediamento. L'esiguità numerica del gruppo di specialisti oltre ad essere materialmente provata dal ristretto gruppo di strutture dove la tecnica importata è applicata, si ritrova anche attestata nelle fonti scritte di poco successive, che descrivono per la Toscana meridionale il passaggio di gruppi di scalpellini, solitamente appartenenti allo stesso nucleo familiare, composti da un numero oscillante tra $\mathrm{i}$ cinque ed i dieci individui ${ }^{40}$. L'individuazione su alcune superfici murarie di pochi graffiti, interpretabili come segni di identità dei diversi maestri lapicidi, proverebbe ulteriormente la ristrettezza numerica del gruppo operativo ${ }^{41}$.

Una simile organizzazione di cantiere, abbiamo visto, fu però possibile nel contesto storico della piena affermazione della signoria territoriale, capace di disporre di risorse

\footnotetext{
39 Il compito di queste maestranze riguardò, oltre l'introduzione di nuove tecniche, anche la stessa progettazione dell'insediamento. Riguardo a questa importante tematica, che esula dall'argomento di questo articolo, si veda BIANCHI 1996 e BIANCHI 2003 b.

40 BIANCHI 1999.

41 BIANCHI 1997.
} 
economiche e soprattutto umane (gli abitanti-costruttori affiancati agli scalpellini) necessarie alle radicali ristrutturazione dei siti fortificati.

Non a caso, in età bassomedievale, tra XIII e XIV secolo, i cambiamenti politici determineranno un'organizzazione del cantiere che risentirà notevolmente delle dinamiche di trasformazione dei vecchi poteri e della affermazione di nuovi.

Nel territorio in esame infatti già dal XII secolo cominciò a farsi sentire sempre più insistentemente l'influenza di Pisa, che si era posta l'obiettivo di acquisire in tempi brevi il pieno controllo dell'area.

Ciò fu ottenuto incoraggiando la nascita dei primi organismi comunali e affiancando le principali cariche con propri funzionari, acquisendo diritti all'interno dei territori castellani ed ottenendo buona parte del controllo dei commerci ${ }^{42}$.

Verso la fine del XIII secolo questa situazione avvenne in parallelo con l'inizio dello spopolamento di un numeroso gruppo di castelli, quasi completamente abbandonati alla fine del secolo successivo ${ }^{43}$. Questi eventi andarono di pari passo con una serie di trasformazioni interne alle diverse famiglie aristocratiche: alcune infatti, definitivamente inubartesi concentrarono i loro interessi nelle politiche cittadine. Altre venute a contrasto proprio con i nuovi poteri pisani scomparirono dalla scena politica.

Tale situazione è piuttosto ben leggibile nell'adozione di particolari tecniche murarie e nella relativa organizzazione di cantiere.

Nei castelli interessati dal graduale processo di spopolamento, gli abitanti infatti continuarono comunque ad avere la necessità di adattare i precedenti spazi alle esigenze abitative del momento, con la creazione di nuovi ambienti, rialzamento di piani, frazionamento di preesistenti spazi interni. Questa serie di operazioni costruttive avvennero quindi senza più lo stretto controllo dell'autorità signorile, disancorate ormai da un ampio progetto edilizio, come era avvenuto nei secoli precedenti.

Il risultato fu il riemergere dei saperi costruttivi degli ambienti tecnici delle differenti comunità. In alcuni casi, come quello del castello di San Silvestro, i nuovi muri furono costruiti con una tecnica, molto simile a quella propria del $\mathrm{x}$ secolo, caratterizzata quindi dall'uso di pietre non lavorate miste però ora ad altre di riuso squadrate, poste con una posa in opera che tra fine XIII secolo e parte di quello successivo divenne sempre più irregolare ${ }^{44}$ (fig. $3 \mathrm{~d}$ ).
Il castello di Donoratico rappresenta anche per questo periodo una sua unicità rispetto ad altri del territorio, dal momento che fu soprattutto nel corso del Duecento che l'assetto abitativo fu completato con lotti di case in pietra e strutture produttive, in contemporanea al massimo potere politico ed economico del ramo dei Della Gherardesca ad esso legato, i conti di Donoratico, che in quegli anni rivestirono importanti cariche nel comune di Pisa. È invece solo nel corso del XIV secolo, quando il castello perse rilevanza per la famiglia che i nuovi interventi legati alle necessità degli abitanti si caratterizzarono per il riutilizzo di pietre squadrate provenienti da strutture più antiche con l'inserimento di zeppe in pietra in filari spesso sdoppiati o con realizzazione di apparecchiature irregolari.

Il passaggio di nuovi saperi legato al costruire si legge, quindi, non più nei centri incastellati ma in quelli trasformati in borghi, provvisti ora di nuovi organismi comunali e soggetti ad un aumento della popolazione, spesso proveniente proprio dai vicini castelli abbandonati.

In questi luoghi, appunto, l'attuale realtà politica, sociale e demografica rese necessaria la costruzione di più ampie cinte in pietra, di palazzi comunali, di case dove alloggiavano gli esponenti di un nuovo ceto medio-alto dedito a nuove attività commerciali.

Nell'edificazione dei circuiti murari di alcuni centri dell'area come Campiglia, Suvereto o Piombino è leggibile, nella comunanza di perimetro racchiuso, come nella tipologia delle porte di accesso, un progetto edilizio unitario che trova riscontro anche nel corredo epigrafico associato a queste strutture che celebrava gli eventi costruttivi. Apparato epigrafico che trova ampi riferimenti nello stile adottato, come nelle formule celebrative, con quello coevo presente a $\mathrm{Pisa}^{45}$. Non a caso i borghi interessati da questo rinnovamento edilizio erano soggetti a Comuni dove le principali cariche venivano assegnate ad esponenti politici provenienti direttamente da Pisa, affiancati a locali investiti di incarichi di minore rilevanza.

Anche le tecniche murarie riflettono questa unitarietà costruttiva che sembrerebbe quindi rimandare ad un flusso di maestranze direttamente provenienti dalla città, impegnate in quest'area maremmana a definire le nuove realtà abitative.

Le caratteristiche delle apparecchiature adottate in più insediamenti si differenziano infatti da quelle del precedente ambiente tecnico di XII secolo in molte caratteristiche: la squadratura della pietra senza perfetti spigoli accompagnata da una saltuaria rifinitura superficiale a subbia; l'utilizzo di

45 Tedeschi 2004; Banti 2000.

42 Ceccarelli 2004.

43 Ceccarelli 2004.

44 BIANCHI 1995. 
conci con dimensioni medie, simili in più centri, con lo sviluppo in particolare della lunghezza rispetto alle altezze dei filari; l'adozione di una posa in opera regolare seppure inframezzata a volte dall'impiego di zeppe in pietra (fig. 3e).

Un così intenso coordinamento di Pisa dell'attività edilizia nei territori ad essa soggetti, non deve del resto stupire, dal momento che alla città si deve la fondazione nella sua area a nord, di alcuni borghi nuovi ${ }^{46}$ e la ricostruzione di strutture urbane preesistenti, dove fu impiegato un gran numero di maestranze, direttamente pagate dal Comune e indirizzate da quest'ultimo nei territori dove era necessario il loro intervento ${ }^{47}$.

Recenti studi sulle architetture della chiesa duecentesca di S.Antimo sopra i Canali a Piombino oltre all'analisi delle ceramiche ritrovate all'interno della volta di copertura dell'abside e nei paramenti esterni della torre campanaria, bene illustrano una organizzazione di cantiere dipendente da Pisa, sia nella scelta delle soluzioni costruttive ed architettoniche sia nei rifornimenti di quella ceramica che sarebbe stata impiegata con funzione strutturale nella stessa volta absidale ${ }^{48}$.

Una volta giunti sul posto però questi specialisti (in realtà più ottimi muratori che buoni scalpellini) non si limitarono solo a lavorare nelle cinte o nell'edificazione dei palazzi comunali e chiese, ma si misero anche al servizio di privati che richiedevano la loro opera.

Il caso di Campiglia, il meglio studiato e più ricco di evidenze materiali rispetto agli altri centri limitrofi, rappresenta uno degli esempi più significativi.

A fronte infatti di una forte influenza di Pisa sia politica che economica, caratterizzata da fitti scambi commerciali, qui, fu anche molto forte l'influenza sociale della città.

Pisa divenne infatti, per i nuovi strati sociali medio alti della comunità campigliese, composti da notai, commercianti, allevatori, medici etc., anche il principale riferimento nella definizione di simboli rispecchianti lo stato acquisito. In questa ottica quindi le architetture delle abitazioni furono una delle più importanti forme di rappresentazione.

Dimenticate ormai le forme tipiche delle residenze signorili interne ai castelli, i profili urbani divennero per gli abitanti di Campiglia la principale fonte di stimoli. Fu così che le maestranze pisane addette alla costruzione degli edifici

\footnotetext{
46 Tra le terre nuove fondate da Pisa in questo territorio ricordiamo S. Vincenzo, BiAnCHi 2000 e Vignale Nuovo costruito alla fine del Duecento dai pisani dopo la distruzione del castello di Vignale Vecchio, CECCARELLI 2004.

47 Informazione personale fornitami da Federico Andreazzoli che ringrazio.

48 Si vedano a proposito i lavori di FiCHERA 2003-2004 e LigUORI 2004-2005.
}

pubblici e delle strutture difensive furono contemporaneamente impegnate ad edificare architetture private identiche a quelle presenti a Pisa, come le case a pilastri insieme a case o botteghe provviste di grandi aperture al piano terreno e bifore ai piani soprastanti, utilizzando la medesima tecnica muraria di quella adottata nei circuiti murari ${ }^{49}$.

E' questa quindi l'ultima importante stagione edilizia all'interno del territorio preso in esame, legata ad una precisa situazione politica che si protrasse sino alla metà del XIV secolo, quando la graduale decadenza politica di Pisa, prima della conquista fiorentina di quest'area, segnò il ritorno ad un'attività costruttiva capillare e frammentata, legata all'iniziativa di singoli committenti e alle conoscenze tecniche più o meno specializzate di piccoli gruppi di costruttori.

\section{Bibliografia}

ANGioni G., 1984, Tecnica e sapere tecnico nel lavoro pre-industriale, La ricerca folklorica, 9, pp. 61-70.

Augenti A., 2000, Dai castra tardoantichi ai castelli del secolo X: il caso della Toscana, in R. Francovich, M. GinATEMPO (a cura di) Castelli. Storia e archeologia del potere nella Toscana medievale, vol. I, Firenze, pp. 25-66.

BALFET H., 1981, Tecnologia, in Il laboratorio dell'etnologo, a cura di R. Creswell, Bologna, pp.63-91.

Banti O., 2000, Monumenta epigraphica Pisana saeculi XV antiquiora, Ospedaletto (Pisa) 2000 («Biblioteca del Bollettino storico pisano. Fonti, 8»).

BELCARI R., 2004, Elementi architettonici e di arredo erratici, in BIANCHI G. (a cura di), Campiglia Marittima: un castello ed il suo territorio. Ricerca storica. Indagini archeologiche, t. 2, pp. 750-760, Firenze.

BelCARi R., BiAnCHI G., FARINELli R., 2003, Il monastero di S. Pietro a Monteverdi. Indagini storico-archeologiche preliminari sui siti di Badiavecchia e Poggio alla Badia (secc. VIII-XIII), in R. Francovich, S. GelichI (a cura di) Monasteri e Castelli tra X e XII secolo. Il caso di $S$. Michele alla Verruca e le altre ricerche storico-archeologiche nella Tuscia occidentale, Firenze, pp. 93-112.

BelCari R., Bianchi G., (a cura di), 2004, Piombino. storia e archeologia di una città dal Medioevo all'età contemporanea, Archeologia dei Paesaggi Medievali - Collana Multimediale, Firenze.

Belli M.-De Luca D.- Grassi F., 2003, Dal villaggio alla formazione del castello: il caso di Rocchette Pannocchieschi, in R. Fiorillo, P. PEDUTO (a cura di), Atti del III Congresso Nazionale di Archeologia Medievale, Salerno 2-5 ottobre 2003, Firenze, pp. 286-291.

BIANCHI G., 1995, L'analisi dell'evoluzione di un sapere tecnico, per una rinnovata interpretazione dell'assetto abitativo e delle strutture edilizie del villaggio fortificato di Rocca S. Silvestro, in Acculturazione e mutamenti. Prospettive nell'archeologia medievale del Mediterraneo, VI ciclo di lezioni sulla ricerca applicata in archeologia. Certosa di Pontignano (SI)Museo di Montelupo (FI), 1-5 marzo 1993, a cura di R. Francovich e E. Boldrini, Firenze, pp. 361-396.

BIANCHI G., 1996 Trasmissione dei saperi tecnici e analisi dei procedimenti costruttivi, Archeologia dell'Architettura, I, pp. 53-65.

BIANCHI G., 1997, Rocca S.Silvestro e Campiglia M.ma: storia parallela di due insediamenti toscani attraverso la lettura delle strutture murarie, in S. Gelichi (a cura di), Atti del I Congresso Nazionale di Archeologia Medievale, Pisa 29-31 maggio 1997, Firenze, pp. 437-444.

49 Per la dettagliata descrizione di queste evidenze si veda BIANCHI 2004e. 
BIANCHI G., 1997, I segni dei tagliatori di pietre negli edifici medievali. Spunti metodologici ed interpretativi, Archeologia dell'Architettura, II, pp. 25-37.

BIANCHI G., 1999, I maestri lombardi nella Toscana centro-meridionale (secc. XII-XV). Indizi documentari ed evidenze materiali, in Magistri d'Europa, Atti del Convegno Internazionale, Como 23-26 ottobre 1996, pp. 140-155.

BiANCHI G., 2000, Indagini archeologiche nella torre di San Vincenzo, Archeologia Medievale, XXVII, pp. 307-314.

BIANCHI G., 2003a, Archeologia dell'architettura e forme di potere tra Xe XIV secolo nella Toscana sud-occidentale: il caso di Campiglia Marittima (LI), Tesi di dottorato in Archeologia Medievale, XV ciclo, Università di Siena, Pisa, Genova, Padova, Venezia.

BiANCHI G., 2003b, Archeologia dell'architettura nei castelli della Toscana sud-occidentale (Val di Cornia-Bassa Val di Cecina. Secc. IX-XII), in R. Fiorillo, P. Peduto (a cura di) Atti del III Congresso Nazionale di Archeologia Medievale, pp. 567-575, Salerno.

BiANCHI G. (a cura di), 2004a, Campiglia Marittima: un castello ed il suo territorio. Ricerca storica. Indagini archeologiche, Firenze.

BiAnCHI G. (a cura di), 2004b, Castello di Donoratico. I risultati delle prime campagne di scavo (2000-2002), Firenze.

BIANCHI G., 2004c, Un castello, un territorio e le sue storie (secc. IX-XVI), sintesi dei dati e considerazioni finali, in BIANCHI G. (a cura di), Campiglia Marittima: un castello ed il suo territorio. Ricerca storica. Indagini archeologiche, t.2, pp. 761-793, Firenze.

BIANCHI G., 2004d, Cronotipologia delle tecniche costruttive, in BIANCHI G. (a cura di), Campiglia Marittima: un castello ed il suo territorio. Ricerca storica. Indagini archeologiche, t. 2, pp. 715-722, Firenze.

BiANCHI G., 2004e, Cronotipologia dell'edilizia abitativa, in BIANCHI G. (a cura di), Campiglia Marittima: un castello ed il suo territorio. Ricerca storica. Indagini archeologiche, t. 2, pp. 723-741, Firenze.

Bianchi G.- Boldrini E.- CitTer C.- Dallai L.- Farinelli R.- FranCovich R.-Grassi F.-Luna A., 1999, Prime indagini a Castel di Pietra (Gavorrano - GR): le campagne 1997-1998, Archeologia Medievale, XXVI, pp. 151-170.

Bogriolo G.P., 1984, Archeologia urbana in Lombardia, Modena

Bogriolo G.P., 1996, Prospettive per l'archeologia dell'architettura, $A r$ cheologia dell'Architettura, I, pp. 11-16.

BOGRIOLO G.P.-GELICHI S., 1998., La città nell'alto medioevo italiano, Bari.

CASINI A., 2004, L'indagine archeologica nel territorio campigliese, in Bianchi G. (a cura di), Campiglia Marittima: un castello ed il suo territorio. Ricerca storica. Indagini archeologiche, t. 2, pp. 141-166, Firenze.

CAmpana S.- Francovich 2003, Landascape archaelogy in Tuscany: cultural resource management, remotely sensed tecniques, GIS based data integration and interpretation, in M. FORTE, P. RAYAN WILLIAMS (a cura di), The reconstruction of archaelogical landscapes through digital tecnologies, BAR series, S1151, pp. 15-18.

CANTINI F., 2003, Il castello di Montarrenti. Lo scavo archeologico (19821987). Per la storia della formazione del villaggio medievale in Toscana (secc. VII-XV), Firenze.

Ceccarelli-Lemut M.L., 1985, La Rocca di San Silvestro nel Medioevo e i suoi signori, in R. FrANCOVICH et alii, Un villaggio di minatori e fonditori di metallo nella Toscana del Medioevo: San Silvestro (Campiglia Marittima), Archeologia Medievale, XII, pp. 322-341.

Ceccarelli-Lemut M.L., 2004, La Maremma populoniese nel Medioevo, in BiAnCHI G. (a cura di), Campiglia Marittima: un castello ed il suo territorio. Ricerca storica. Indagini archeologiche, t. 1, pp. 1-116, Firenze.

COLLAVINI S., 1998, "Honorabilis domus et spetiossimus comitatus». Gli Aldobrandeschi da "conti» a "principi territoriali» (secoli IX-XIII), Pisa.

CUTERI F., 1990, Recenti indagini a Suvereto (Livorno): un contributo toscano all'archeologia dei centri storici (minori), Rassegna di Archeologia, 9, pp. 431-464.

DAllai L., 2000, Opifici metallurgici sul promontorio di Piombino: primi dati topografici, in G.P. Brogiolo (a cura di), Atti del II Congresso
Nazionale di Archeologia Medievale, Brescia 28 settembre-1 ottobre 2000, Firenze, pp. 3194-198.

Dallai L.-FarinElli R., 1998, Castel di Pietra e l'Alta Valle del Bruna. Indagini storiche e topografiche per la redazione di una carta archeologica, Archeologia Medievale, XXV, 1998, pp. 49-74.

FARINELli F., 2000, I castelli nei territori diocesani di Populonia-Massa e Roselle-Grosseto (secc. X-XIV), FranCOVICH R.-GINATEMPO M. (a cura di) 2000, Castelli. Storia e archeologia del potere nella Toscana medievale, vol. I, Firenze, pp. 239-284.

FARINELli R., 2003, I castelli nella Toscana delle città «deboli». Dinamiche insediative e potere rurale nella Toscana meridionale (secoli VII-XIV), Tesi di dottorato in Archeologia Medievale, XV ciclo Università di Siena, Pisa, Genova, Padova, Venezia.

FARINELli R.-FrANCOVICH R.2000 (a cura di), Guida alla Maremma medievale. Itinerari di archeologia nella provincia di Grosseto, Siena.

Fichera G., 2003-2004, Archeologia dell'Architettura di un edificio ecclesiastico: la chiesa di Sant'Antimo sopra i Canali a Piombino (LI), Tesi di Laurea, Facoltà di Lettere e Filosofia, Università degli Studi di Siena, relatore Riccardo Francovich, correl. Giovanna Bianchi.

Francovich R.1991, Rocca San Silvestro, Roma.

FRANCOVICH R., 2002, Changing structures of settlements in early Medieval Italy, in C. LA RocCA (a cura di) Italy in the early middle ages, «Short Oxford History of Italy», OUP, Oxford, pp144-167.

FranCOVICH R.-WICKHAM C., 1994, Uno scavo archeologico ed il problema dello sviluppo della signoria territoriale: Rocca San Silvestro e i rapporti di produzione minerari, Archeologia Medievale, XXI, pp. 7-30.

Francovich R., Citter C. Goracci M., Farinelli R., 2000, Castel di Pietra e la diga dei Muracci. Rapporto preliminare delle ricerche 1999, in G.P. Brogiolo (a cura di), Atti del II Congresso Nazionale di Archeologia Medievale, Brescia 28 settembre-1 ottobre 2000, Firenze, pp. 189-193.

Francovich R.- Hodges R., 2003, Villa to village. The transformation of the Roman Countryside in Italy, c.400-1000, Duckworth, London.

FrANCOVICH R.- BIANCHI G., 2003, L'archeologia dell'elevato come archeologia, Arqueologia de la Arquitectura, I, pp.

FranCOVICH R.-Gelichi S. c.s. (a cura di), Il monastero di S. Quirico a Populonia. I risultati delle prime campagne di scavo, Rassegna di Archeologia.

GAlETTI P., 1998, Abitare nel Medioevo. Forme e vicende dell'insediamento rurale nell'Italia altomedievale, Firenze.

GRASSI F., 2004, I reperti ceramici, conclusioni, in BIANCHI G. (a cura di), Campiglia Marittima: un castello ed il suo territorio. Ricerca storica. Indagini archeologiche, t. 2, pp. 342-361, Firenze.

La CeCla F., 1996, Mente locale. Per un'antropologia dell'abitare, Milano.

LEROI-GOURHAN A., 1971, L'homme et la matière, Parigi.

Levy F.-Segaud M., 1983, Anthropologie de l'Espace, Parigi, 1983.

LiguORI S., 2004-2005, Il riempimento della volta di S. Antimo sopra $i$ Canali a Piombino: nuove acquisizioni sulla maiolica arcaica pisana e la ceramica di importazione di XIII secolo, Tesi di Laurea, Facoltà di Lettere e Filosofia, Università degli Studi di Siena, relatore Riccardo Francovich, correl. Graziella Berti, Giovanna Bianchi.

Mannoni T.- Giannichedda E., 1996, Archeologia della produzione, Torino.

MannONi T., 1988, Archeologia della produzione, in Francovich R.-PARENTI R., 1988 (a cura di), Archeologia e Restauro dei monumenti. I ciclo di lezioni sulla ricerca applicata in archeologia. Certosa di Pontignano (Siena) 28 settembre-10 ottobre 1987, Firenze, pp. 403-420.

QUiRÓS CASTILlo J.A., 1998, La silleria y las tecnicas constructivas medievales: hisotria social y tecnica de la prodiccion arquitectonica, $\mathrm{Ar}$ cheologia Medievale, XXV, pp. 235-246.

Quirós Castillo J. A., 1999, La Valdinievole nel medioevo. "Incastellamento" e archeologia del potere nei secoli X-XII, Pisa.

TEDESCHI C., 2004, Le epigrafi dell'edilizia civile (secoli XIII-XIV), in BIANCHI G. (a cura di), Campiglia Marittima: un castello ed il suo territorio. Ricerca storica. Indagini archeologiche, t. 2, pp. 742-749, Firenze. 
TECNICHE COSTRUTTIVE E STRUTTURE DI POTERE NELLA TOSCANA SUD-OCCIDENTALE (SECC. VIII-XIV)

Valenti M. (a cura di) 1996, Poggio Imperiale a Poggibonsi: dal villaggio di capanne al castello in pietra. I. Diagnostica archeologica e campagne di scavo 1991-1994, Firenze.

VAlENTi M., 1999, Carta archeologica della provincia di Siena, vol. III. La Val d'Elsa, Siena.

VALENTI M., 2004, L'insediamento altomedievale nelle campagne toscane. Paesaggi, popolamento e villaggi tra VI eX secolo, Firenze.

VOLPE G., 1964, Toscana Medievale. Massa marittima, Volterra e Sarzana, Firenze. 